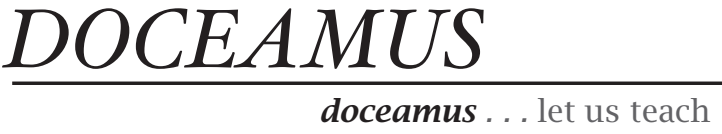

\section{History of Mathematics: Seeking Truth and Inspiring Students}

\section{Alex M. McAllister and Diana White}

Our doctoral programs helped us master the research methodologies and disciplinary norms of our respective fields in pure mathematics. However, as professors, we both developed a strong desire to deepen our knowledge of the history of mathematics and its research methodologies. In this column, we provide a brief overview, anchored in concrete examples and based on our experiences, of how learning more about the history of mathematics as well as the basics of historiography can benefit working mathematicians professionally.

\section{Rigorous Research}

As mathematicians, we base a great deal of our work on deductive reasoning from definitions and axioms. As a result, we may adopt a skeptical view toward disciplines that we perceive as lacking the same "rigor." We might also view historical

Alex M. McAllister is professor of mathematics at Centre College. His email address is a 1 ex. mca11 i ster@centre. edu.

Diana White is associate professor of mathematics at the University of Colorado Denver. Her email address is Diana. Whi te@ucdenver . edu.

The authors first learned about many of these ideas from the two-day MAA Short Course: Reading, Writing, Doing the History of Mathematics: Learning the Methods of Historical Research, just before the 2014 Joint Mathematics Meetings.

Members of the Editorial Board for Doceamus are: David Bressoud, Roger Howe, Karen King, William McCallum, and Mark Saul.

DOI: http://dx.doi.org/10.1090/noti1207 research as simply creating lists of people, papers, accomplishments, and inventions. Yet the history of mathematics is so much more than this!

In addition to describing the development of mathematics, historians of mathematics seek to understand the influence of ambient culture, social conventions and norms, and mathematicians as individuals and in relationships with others. They care about the extramathematical work and interests of mathematicians, because these often provide the broader context of and influences on their work. A robust understanding of history is developed through a dynamic process of "zooming in" on specific details and "zooming out" to a broader perspective of contemporary cultural, social, political, and scientific influences. Historical research produces reliable, accurate historical knowledge via methodologies that, while different, are every bit as sophisticated and well developed as the practices of mathematicians and scientists.

\section{Historical Accuracy and Contextual Understanding}

As students and later as professors, we typically become familiar with a variety of historical anecdotes related to mathematics, which we may in turn repeat to our students or others. However, because of our lack of expertise in the history of mathematics, we are usually unable to evaluate whether these anecdotes are true or mere apocryphal tales.

The tales of mathematics involve more than just the mathematics itself. Mathematical anecdotes are perhaps akin to family gossip, capitalizing on humans' innate love of sharing stories. Students often 
benefit from this humanizing of mathematics and can come to perceive mathematics as a dynamic evolving field of study and inquiry rather than just some static, staid collection of facts. At the same time, though, each story we tell should be true rather than hagiography or a sort of false fishing tale about "the one that got away." As mathematicians we aspire to share mathematical truths with our students, and this goal should also motivate our fidelity to accurately representing the history of mathematics.

Some scholars, especially novices, have a tendency to read modern results and perspectives into the writings and results of preceding generations of mathematicians. For example, the geometric results found in Euclid's Elements can be interpreted and explained in terms of algebra. A modern mathematician might take this algebraic interpretation based on current techniques as evidence that the Greeks knew algebra. This observation might lead them to mistakenly posit that Elements is a geometric garland covering the underlying algebra rather than acknowledge its true nature as a synthesis of the state-of-the-art in geometry.

Historians strive to adopt the unique perspectives of the mathematicians from each time, place, and social context that is being studied. Babylonian, Greek, Chinese, Indian, Islamic, European, and modern mathematicians all explored different questions, developed distinct approaches for grappling with these questions, and varied in their perceived need for and rigor of mathematical arguments. In short, historians must be careful to investigate mathematical developments in their appropriate context to avoid creating a "whig" history of mathematics which presents the past as an inevitable progression toward the more enlightened present while ignoring mathematical pathways that are not part of our current methodologies.

\section{Effective Teaching Using the History of Mathematics}

The history of mathematics can inform our approach to teaching entire fields of mathematics or specific topics within a particular field. For the former, a prime example is Otto Toeplitz's Genetic Approach to Calculus, which presents the ideas of calculus from a historical perspective of ideas evolving over centuries by examining the particular results of Archimedes, Kepler, Galileo, Fermat, and culminating with Newton and Leibniz. For the latter, we discuss an example shared by Fred Rickey at one of last year's Joint Mathematics Meetings' minicourses (1). He presents his Real Analysis class with Cauchy's "proof" that if a series of continuous functions converges, then the series converges to a continuous function. For homework, his students explore counterexamples to this supposed "theorem." During the next class period, after his students express their frustration with the "false proof," Rickey helps them understand the error in Cauchy's proof and then develops the notion of uniform convergence.

Studying the history of mathematics can also help students understand and embrace mathematical traditions. For example, the notions of proof and rigor have had different meanings in various times and cultures. In the fourth century BCE, Euclid provided a deductive proof of the Pythagorean Theorem (in Elements, see also Liu Hui's annotated version of Jiu zhang suan shu Nine Chapters on the Mathematical Art) from 263 CE provided a "proof by picture" of this result that was held in similarly high regard by his peers. Approaching the modern era, in 1899 CE, David Hilbert introduced a "more rigorous" development of Euclidean geometry in his Grundlagen der Geometrie. A comparison of these various proofs provides a wonderful, focused opportunity for students to discuss the evolving nature of proof itself.

We regularly create and teach proofs by induction. Yet many prior generations of mathematicians were content with using some variation of the phrase "and so on" when it appeared clear that the arguments would continue indefinitely in the same fashion. For example, Euclid's original proof that there exist infinitely many primes considers only the case of there being exactly three primes. We now explain "his" proof with the supposition that there exists some arbitrary, finite number of primes, but in Euclid's time and for centuries afterwards, his original proof was regarded as sufficient and rigorous. Our students can better understand the contemporary notion of rigorous proof when we contrast modern proofs with the arguments of our mathematical ancestors.

\section{Resources for Deepening Knowledge}

Just as in mathematical research, the quest for new historical knowledge can require great persistence. Some good starting places include the Dictionary of Scientific Biography (2) and the MacTutor History of Mathematics archive (3), both of which contain references to original sources for further study. A historian must go deeper, though, and examine extant, original manuscripts housed in archives. Fortunately, libraries continue scanning their archival materials and providing access through the Web. In parallel, translators are making an increasing number of manuscripts available in diverse languages, although translations can present their own challenges in their accuracy and reliability.

The Mathematical Association of America's History of Mathematics Special Interest Group (HOMSIGMAA) provides many resources on their website (4). These links include references for mathematicians interested in the basics of reading, writing, and doing the history of mathematics. Another starting place might be attending a talk on the history of mathematics at the next Joint Math 


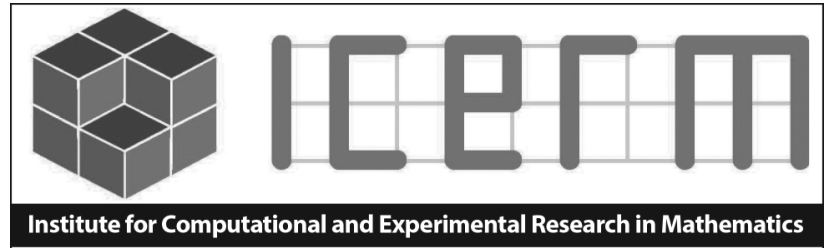

SUMMER TOPICAL WORKSHOPS

Integrability in Mechanics and Geometry: Theory and Computations / June 1-5, 2015

Organizers: A. Calini (College of Charleston), B. Khesin (Univ. of Toronto), G. Mari-Beffa (Univ. of Wisconsin), V. Zharnitsky (Univ. of Illinois at Urbana-Champaign)

Description: This workshop will focus on topics at the interface of classical mechanics, differential geometry, and computer experiments. The directions of current research to be explored include the study of invariants and complete integrability of geometrically motivated differential equations, sub-Riemannian geometry, geometric control, nonholonomic systems, and computational methods in mechanics and dynamics.

\section{Computational Geometric Topology in Arrangement Theory / July 6-10, 2015}

Organizers: N. Budur (KU Leuven and University of Notre Dame), G. Denham (Univ. Western Ontario), A. D. Macinic (IMAR, Bucharest), D. Matei (IMAR, Bucharest), L. Maxim (UW-Madison), H. Schenck (UIUC), M. Wakefield (US Naval Academy)

Description: This workshop will bring together mathematicians working on combinatorial, geometric and topological properties of arrangements. In addition to fundamental open problems in the area, participants will explore connections to tropical geometry, configuration spaces, and applications, building bridges between those working on different aspects of the area.

\section{Computational and Analytical Aspects of Image Reconstruction / July 13-17, 2015}

Organizers: G. Ambartsoumian (Univ. of Texas), V. Druskin (Schlumberger-Doll), E. Klann (Johannes Kepler Univ.), V. P. Krishnan (TIFR Centre for Applicable Mathematics), A. Louis (Universität des Saarlandes), and E. T. Quinto (Tufts Univ.)

Description: The mathematical study of image reconstruction problems can have a huge impact on human life. More efficient mathematical algorithms for X-ray tomography and more accurate mathematical models in seismic or hybrid imaging can lead to better imaging devices in fields such as medicine and remote sensing. This workshop will bring together experts working in computational and analytical aspects of image reconstruction.

\section{Program and participant details:} icerm.brown.edu

ICERM welcomes applications for long- and short-term visitors. Support for local expenses may be provided. Decisions about online workshop applications are typically made 1-3 months before each program, as space and funding permit. ICERM encourages women and members of underrepresented minorities to apply.
About ICERM: The Institute for Computational and Experimental Research in Mathematics is a National Science Foundation Mathematics Institute at Brown University in Providence, RI.

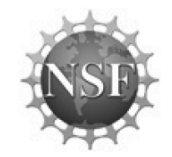

Meetings or at MathFest. Exploring the history of mathematics through these various resources enables us to grow professionally and to come to think differently about our profession and our mathematical ancestors.

\section{Final Thoughts}

Our doctoral programs provide solid training for mathematical research, and increasingly our profession augments this learning with preparation for teaching. We propose an additional step in our ongoing professional development both as teachers and scholars: learning how our discipline evolved into our contemporary study of mathematics as well as how historical research is conducted.

The history of mathematics can inform our understanding of basic topics, strengthen our teaching, and help us better understand our place in the overall human story of mathematics. Not only will we grow as mathematicians, we will also build our capacity to help our students understand mathematics as a human endeavor, developing in response to and driving various aspects of society. Some of us may even decide to go beyond being consumers of the history of mathematics and to learn the in-depth research procedures of historiography, making original contributions to this field.

\section{References}

[1] Fred Rickey at fredrickey. info/shared this example at the MAA Short Course: Reading, Writing, Doing the History of Mathematics: Learning the Methods of Historical Research, just before the 2014 Joint Mathematics Meetings.

[2] Dictionary of Scientific Biography at hi storyofmathematics.org.

[3] MacTutor History of Mathematics archive at wwwhistory.mcs.st-andrews.ac.uk/

[4] HOMSIGMAA at historyofmathematics.org/. 